\title{
A NON-SUBSTITUTION THEOREM WITH HETEROGENEOUS LABOR
}

\author{
Adolfo García de la Sienra* \\ Facultad de Economía, Universidad Veracruzana
}

(Received 12 august 2001, accepted 10 december 2001)

\begin{abstract}
The present paper shows that the Non-Substitution Theorem is actually constituted by two statements. The first one asserts that it is possible to find a finite number of production processes, one in each branch of the economy, in such a way that the elements of the convex cone spanned by such activities are all efficient. The second one asserts that it is possible to find a finite number of production processes, one in each branch of the economy, such that the set of efficient processes is contained in the convex cone spanned by such processes. The first statement is proven within more general structures than that of Leontief. The second one is proven within a modification of such structures that admits heterogeneous labor.
\end{abstract}

\section{Resumen}

El presente artículo muestra que el teorema de no sustitución consta en realidad de dos proposiciones. La primera afirma que es posible encontrar un número finito de procesos de producción, uno en cada rama de la economía, de tal manera que todos los elementos del cono convexo generado por dichas actividades son eficientes. La segunda afirma que es posible encontrar un número finito de procesos de producción, uno en cada rama de la economía, de tal manera que el conjunto de los procesos eficientes está contenido en el cono generado por dichos procesos. La primera aserción se demuestra en estructuras más generales que las de Leontief. La segunda se demuestra en una modificación de dichas estructuras que admite trabajo heterogéneo.

JEL Classification: D24.

Keywords: Production, and Factors.

* Facultad de Economía, Universidad Veracruzana, Av. Xalapa y Manuel Ávila Camacho, 91020 Xalapa, Veracruz, E-mail: sienra@dino.uv.mx.

The author wishes to thank two anonymous referees of this journal for valuable guidance and suggestions. Moreover, the author gratefully acknowledges the useful comments and suggestions made upon a previous version of the present paper by Professor Francisco VenegasMartínez. This paper was written with support of CONACyT project $28630-\mathrm{H}$. 


\section{Introduction}

A Non-Substitution Theorem asserts, essentially, that rational producers cannot help but choosing a certain technology because that technology is the only efficient one. The Non-Substitution Theorem for a static Leontief economy was proven independently for the first time by Samuelson (1951) and GeorgescuRoegen (1967), by means of calculus techniques. Using more general techniques, the theorem was proven by Koopmans (1951) for the case of three industries, but the most perspicuous formulation and proof of this result is due to Arrow (1951). Levahri (1965) provides another proof of the theorem showing that, even though a producer may shift from one activity to another, and back to the first, as the interest rate changes in the same direction, that is not possible for the productive system as a whole. Mirrles (1969) proved the theorem for a dynamic economy which excludes joint production. Stiglitz (1970) admits durable goods within a dynamic economy in balanced growth. One of the aims of the present paper is to prove a (static) Non-Substitution Theorem with heterogeneous labor.

The Non-Substitution Theorem is sometimes presented as an assertion about profit rates and price systems: under certain conditions, a given profit rate determines uniquely an equilibrium price system for the economy. Yet, this assertion is a logical consequence of a property that economies have under such conditions, to wit, that it is possible to represent the set of all its efficient feasible production processes as a convex polyhedral cone spanned by a finite set of basic activities. Hence, there is a number of assertions logically related to the Non-Substitution Theorem.

In order to provide a precise formulation of the theorem, suppose that there are $\eta$ producers in the economy, labeled $1, \ldots, \eta$, with production possibility sets $X_{1}, \ldots, X_{\eta}$, respectively, as characterized by the following definition.

Definition 1: $\mathcal{X}$ is a Leontief productive structure if there are positive integers $\eta, \nu$ and $\mu$ such that:

(1) $\mathcal{X}=\left\langle X_{1}, \ldots, X_{\eta}\right\rangle$;

(2) $X_{h}$ is a closed convex cone in the linear space $\mathbb{R}^{\nu+2 \mu}$;

(3) Any element of $X_{h}$ is a non-negative vector of the form $[\mathbf{x}, \underline{\mathbf{x}}, \overline{\mathbf{x}}]$, where the $\nu$-dimensional vector $\mathbf{x}$ is intended to represent the expenditures of labor of the process, the $\mu$-dimensional vector $\underline{x}$ stands for the inputs, and the $\mu$-dimensional vector $\overline{\mathbf{x}}$ denotes the outputs. Thus, the elements of $X_{h}$ are production processes in the stock version;

(4) Labor is homogeneous; i.e., $\mathbf{x}$ is a one-dimensional vector $x$ (a scalar).

(5) There is no joint production; i.e., $\mu=\eta$ and $\bar{x}_{m h} \neq 0$ only if $m=h$, for $m=1, \ldots, \mu$ and any process $[\mathbf{x}, \underline{\mathbf{x}}, \overline{\mathbf{x}}] \in X_{h} ;$

(6) Labor is indispensable; i.e., $\mathbf{x}=\mathbf{0}$ implies $\underline{\mathbf{x}}=\underline{\mathbf{0}}$ and $\overline{\mathbf{x}}=\overline{\mathbf{0}}$ for any process $[\mathrm{x}, \underline{\mathbf{x}}, \overline{\mathrm{x}}] \in X_{h}$

(7) Labor is productive; i.e., $\underline{x}=\underline{0}$ or $\bar{x}=\overline{0}$ implies $\mathrm{x}=0$ for any process $[\mathrm{x}$, $\underline{\mathbf{x}}, \overline{\mathrm{x}}] \in X_{h}$. 
Vector $\widehat{\mathbf{x}}=\overline{\mathbf{x}}-\underline{\mathbf{x}}$ represents the net outputs of process $[\mathbf{x}, \underline{\mathbf{x}}, \overline{\mathbf{x}}]$. The set $X=\sum_{h} X_{h}$ is called the global production possibility set and its members are called global processes. The set of all processes in $X$ having non-negative net outputs shall be denoted by $X^{+}$. The netput form or flow version of process $\widetilde{\mathrm{x}}=[\mathrm{x}, \underline{\mathrm{x}}, \overline{\mathrm{x}}]$ is $\breve{\mathrm{x}}=[-\mathbf{x}, \widehat{\mathrm{x}}]$.

Definition 2: Let $Y$ be a set of production processes, and $\widetilde{\mathbf{y}}$ and $\widetilde{\mathbf{y}}^{\prime}$ elements of $Y$.

(1) Process $\tilde{\mathbf{y}}$ is more efficient than process $\widetilde{\mathbf{y}}^{\prime}$ iff $\breve{\mathbf{y}} \geq \breve{\mathbf{y}}^{\prime}$. It is strictly more efficient iff $\breve{\mathbf{y}} \geq \breve{\mathbf{y}}^{\prime}$. A process $\widetilde{\mathbf{y}} \in Y$ is efficient iff there is no process in $Y$ strictly more efficient than $\widetilde{\mathbf{y}}$.

(2) $Y$ is productive iff $Y^{+}$contains a process $\widetilde{\mathbf{y}}$ such that $\widehat{\mathbf{y}}>\widehat{\mathbf{0}}$.

If we let $H$ be the set $\{1, \ldots, \eta\}$, the Non-Substitution Theorem itself can be formulated as follows. ${ }^{1}$

The Non-Substitution Theorem: In a Leontief productive structure, let $E$ be the set of efficient processes of $X$. If $X^{+}$is productive, there is a finite number of activities $\widetilde{\mathbf{x}}_{1}, \ldots, \widetilde{\mathbf{x}}_{\eta}$ with $\widetilde{\mathbf{x}}_{h} \in X_{h}$ for every $h \in H$ such that the cone spanned by these processes, $K\left(\widetilde{\mathbf{x}}_{1}, \ldots, \widetilde{\mathbf{x}}_{\eta}\right)$, is precisely $E$. Moreover, for every final demand vector $\mathbf{z} \geq 0$, there is a non-negative amount of labor $z$ such that $[z, \mathbf{z}] \in E$.

Thus, it is easy to see that the theorem is actually a conjunction of three different propositions:

Proposition 1: There is a finite number of activities $\widetilde{\mathbf{x}}_{1}, \ldots, \widetilde{\mathbf{x}}_{\eta}$, with $\widetilde{\mathbf{x}}_{h} \in X_{h}$ for $h \in H$, such that $K\left(\widetilde{\mathbf{x}}_{1}, \ldots, \widetilde{\mathbf{x}}_{\eta}\right) \subseteq E$.

Proposition 2: There is a finite number of activities $\widetilde{\mathbf{x}}_{1}, \ldots, \widetilde{\mathbf{x}}_{\eta}$, with $\widetilde{\mathbf{x}}_{h} \in X_{h}$ for $h \in H$, such that $E \subseteq K\left(\widetilde{\mathbf{x}}_{1}, \ldots, \widetilde{\mathbf{x}}_{\eta}\right)$.

Proposition 3: Every non-negative demand vector can be exactly produced by some efficient process.

The meaning of Proposition 1 is that processes from all production branches can be chosen in such a way that any non-negative linear combination of these processes is efficient. Proposition 1 is important for the foundations of the labor theory of value as understood by Ulrich Krause and myself, ${ }^{2}$ and so it has direct relevance for economic theory.

The import of Proposition 2 is that there is a finite number of activities such that each efficient process can be expressed as linear combination of these

1 See Arrow (1951), pp. 158, 164, and Nikaido (1968), pp. 190-191.

2 To see the importance of Proposition 1 for this theory see García de la Sienra (1992) and (1996). 
activities. The properly non-substitutional statement is Proposition 2, which is usually coupled with Proposition 3, because Proposition 3 is also a consequence of the assumptions supporting Proposition 2. Naturally, this proposition is also important for the theory of value and provides a foundation for the general theory of Leontief systems with homogeneous labor.

My first goal in this paper is to prove Proposition 1 under assumptions that are more general than those defining Leontief productive structures. A second goal is to prove also Proposition 2, under slightly different assumptions than those represented by the axioms of Definition 1, allowing heterogeneous labor. This is done in section 2. Finally, in section 3, I discuss the import and limitations of the version of the Non-Substitution Theorem here introduced, as well as the open problems left for further research.

\section{Productive Structures}

I shall define a type of structures representing productive structures of more general economies. What I intend to model by means of this mathematical framework is the group of "producers", understood as the different "branches" of the economy. In the usual Leontief setting, each "producer" has technologies available to produce only one kind of good, but in a setting allowing joint production that requirement is dropped.

Definition 3: $\mathcal{X}$ is productive structure iff there are positive integers $\eta, \nu$ and $\mu$ such that:

(1) $\mathcal{X}=\left\langle X_{1}, \ldots, X_{\eta}\right\rangle$;

(2) $X_{h}$ is a closed convex cone in the linear space $\mathbb{R}^{\nu+2 \mu}$;

(3) Any element of $X_{h}$ is a non-negative vector of the form $[\mathbf{x}, \underline{\mathbf{x}}, \overline{\mathbf{x}}]$, where the $\nu$-dimensional vector $\mathbf{x}$ is intended to represent the expenditures of labor of the process, the $\mu$-dimensional vector $\underline{\mathbf{x}}$ stands for the inputs, and the $\mu$-dimensional vector $\overline{\mathbf{x}}$ denotes the outputs. Thus, the elements of $X_{h}$ are production processes in the stock version;

(4) Labor is indispensable;

(5) Labor is productive;

Notice that the Leontief productive structure is a special case of the productive structure. The definitions of $X, X^{+}$and $E$ are analogous to those already given. An interesting fact is that for every process in $X^{+}$there is always an efficient process that produces at least the same netput as the first one with the same or even less amount of labor. This is the meaning of the following lemma.

Lemma 1: Let $X^{+}$be the set of global processes with non-negative net outputs of a productive structure $X$. Then, for every process $\widetilde{\mathbf{x}} \in X^{+}$there is an efficient process $\widetilde{\mathbf{y}}^{*} \in X^{+}$such that $\widehat{\mathbf{y}}^{*} \geq \widehat{\mathbf{x}}$ and $\mathbf{y}^{*} \leq \mathbf{x}$. 
Proof: The strategy of the proof is simple. I will show, first, that the set

$$
E(\widetilde{\mathbf{x}})=\{\widetilde{\mathbf{y}} \mid \widehat{\mathbf{y}} \geq \widehat{\mathbf{x}} \text { and } \mathbf{y} \leq \mathbf{x}\}
$$

is compact and, second, I will use the fact that the function $\varphi: E(\widetilde{\mathbf{x}}) \rightarrow \mathbb{R}$, that assigns to every vector $\widetilde{\mathbf{y}} \in E(\widetilde{\mathbf{x}})$ the number

$$
\left(\widehat{y}_{1}+\cdots+\widehat{y}_{\mu}\right)-\left(y_{1}+\cdots+y_{\nu}\right)
$$

is continuous: it will follow (by Weierstrass Theorem) that $\varphi$ reaches a maximum at $\widetilde{\mathbf{y}}^{*}$. It will be easy to see that $\widetilde{\mathbf{y}}^{*}$ is an efficient point in $X^{+}$. Since $\widetilde{\mathbf{0}} \in X^{+}$, in order to prove that $E(\widetilde{\mathbf{x}})$ is bounded notice that the set

$$
F=\{\mathbf{y} \mid[\mathbf{y}, \underline{\mathbf{y}}, \overline{\mathbf{y}}] \in E(\widetilde{\mathbf{x}})\}
$$

is bounded (because $\mathbf{0} \leq \mathbf{y} \leq \mathbf{x}$ for every $[\mathbf{y}, \mathbf{y}, \overline{\mathbf{y}}] \in E(\widetilde{\mathbf{x}})$ ). Now, if $E(\widetilde{\mathbf{x}})$ were unbounded, there would be a sequence $\left(\widetilde{\mathbf{y}}_{k}\right)$ in $E(\widetilde{\mathbf{x}})$ such that $\left(\left\|\widetilde{\mathbf{y}}_{k}\right\|\right)$ is increasing and unbounded. Nevertheless, the corresponding sequence of labor input vectors $\left(\mathrm{y}_{k}\right)$, can be seen to converge to a limit $\mathbf{y}$ (not necessarily in $F$ ) because $F$ is bounded. Let

$$
\widetilde{\mathbf{z}}_{k}=\left(\left\|\widetilde{\mathbf{y}}_{k}\right\|+1\right)^{-1} \widetilde{\mathbf{y}}_{k} .
$$

Since $X^{+}$is a cone, $\widetilde{\mathbf{z}}_{k} \in X^{+}$. Moreover, $\left(\widetilde{\mathbf{z}}_{k}\right)$ is bounded because $\left\|\widetilde{\mathbf{z}}_{k}\right\| \leq 1$. Hence, without loss of generality, we may assert that (a subsequence of) ( $\widetilde{\mathbf{z}}_{k}$ ) converges to a point which must belong to $X^{+}$because $X^{+}$is closed. Since $\left(\left\|\widetilde{\mathbf{z}}_{k}\right\|\right) \rightarrow 1, \widetilde{\mathbf{z}} \neq \widetilde{\mathbf{0}}$ and so, due to the productivity and indispensability of labor, $\overline{\mathbf{z}} \geq \overline{\mathbf{0}}$. On the other hand, since $\left(\mathbf{y}_{k}\right) \rightarrow \mathbf{y}$ as $k \rightarrow \infty$,

$$
\begin{aligned}
\mathbf{z} & =\lim _{k \rightarrow \infty} \mathbf{z}_{k} \\
& =\lim _{k \rightarrow \infty}\left(\left\|\widetilde{\mathbf{y}}_{k}\right\|+1\right)^{-1} \cdot \lim _{k \rightarrow \infty} \mathbf{y}_{k} \\
& =0 \cdot \mathbf{y} \\
& =0 .
\end{aligned}
$$

Hence, given that labor is indispensable, $\underline{z}=\overline{0}$. This contradiction shows that $E(\widetilde{\mathbf{x}})$ is bounded.

Now, it is easy to see that $E(\widetilde{\mathbf{x}})$ is closed, because the limit of any convergent sequence of points of $E(\widetilde{\mathbf{x}})$ is in $X^{+}$, which is closed, and also satisfies the condition for belonging to $E(\widetilde{\mathbf{x}})$.

Clearly, since $\varphi$ is continuous, it has a maximum at a point $\widetilde{\mathbf{y}}^{*}$ in $E(\widetilde{\mathbf{x}})$. I claim that this point is actually efficient in $X^{+}$. For suppose, on the contrary, that there is a $\widetilde{\mathbf{y}}^{* *}$ that is strictly more efficient than $\widetilde{\mathbf{y}}^{*}$. Then, $\varphi\left(\widetilde{\mathbf{y}}^{* *}\right)>\varphi\left(\widetilde{\mathbf{y}}^{*}\right)$, which is impossible because $\varphi$ had reached a maximum at $\widetilde{\mathbf{y}}^{*}$. 


\section{Efficiency and Non-Substitution}

Probably due to its outstanding implications regarding the uniqueness of a price vector for the economy, the Non-Substitution Theorem has received a great deal of attention. Yet, the weaker assertion labeled here as Theorem 1 has also important implications for the existence of shadow prices. As it is shown in García de la Sienra (1996), any productive structure in which conditions of Theorem 1 hold has, at least, one system of prices and wages $(\mathbf{p}, \mathbf{w})$ such that, for any netput $\widehat{\mathbf{x}}$, the value of netput $\widehat{\mathbf{x}}$ at these prices, $\mathbf{p} \widehat{\mathbf{x}}$, is equal to the wages paid to the labor power that produced this netput, wx. What the NonSubstitution Theorem adds is that this pair $(\mathbf{p}, \mathbf{w})$ is unique up to similarity transformations (multiplication by a scalar).

Theorem 1: There is a finite number of basic activities such that every nonnegative linear combination of these basic activities is efficient; $i, e, \exists\left(\widetilde{\mathbf{x}}_{1}, \ldots \widetilde{\mathbf{x}}_{\eta}\right)$ $\in X_{1} \times \cdots \times X_{\eta}: K\left(\widetilde{\mathbf{x}}_{1}, \ldots, \widetilde{\mathbf{x}}_{\eta}\right) \subseteq E$, where $K\left(\widetilde{\mathbf{x}}_{1}, \ldots, \widetilde{\mathbf{x}}_{\eta}\right)$ is the convex polyhedral cone spanned by processes $\widetilde{\mathbf{x}}_{1}, \ldots, \widetilde{\mathbf{x}}_{\eta}$.

Proof: Let $\widetilde{\mathbf{x}}$ be an efficient global process with $\widehat{\mathbf{x}}>\widehat{0}$ and let $\left\{\widetilde{\mathbf{x}}_{h}\right\}$ be a family of basic processes such that $\widetilde{\mathbf{x}}=\sum_{h} \widetilde{\mathrm{x}}_{h}$. I will show that the convex cone $K\left(\widetilde{\mathbf{x}}_{1}, \ldots, \widetilde{\mathbf{x}}_{\eta}\right)$ spanned by these processes is a set of efficient activities. Let

$$
\mathbf{L}=\left(\begin{array}{ccc}
x_{11} & \cdots & x_{1 \eta} \\
\vdots & \ddots & \vdots \\
x_{\nu 1} & \cdots & x_{\nu \eta}
\end{array}\right)
$$

and

$$
\mathbf{N}=\left(\begin{array}{ccc}
\widehat{x}_{11} & \cdots & \widehat{x}_{1 \eta} \\
\vdots & \ddots & \vdots \\
\widehat{x}_{\mu 1} & \cdots & \widehat{x}_{\mu \eta}
\end{array}\right)
$$

where the $h$ th column of $\mathrm{L}$ is the vector of labor inputs of activity $\widetilde{\mathbf{x}}$, and the $h$ th column of $\mathbf{N}$ is that of net outputs. Hence, $\breve{\mathbf{x}}=[-\mathbf{L t}, \mathbf{N t}]$ for some positive state vector $t$.

Let $\widetilde{\mathbf{y}}$ be an arbitrary element of $K\left(\widetilde{\mathbf{x}}_{1}, \ldots, \widetilde{\mathbf{x}}_{\eta}\right)$, so that $\breve{\mathbf{y}}=[\mathbf{L s}, \mathbf{N s}]$ for some non-negative $\mathbf{s}$, and suppose that $\widetilde{\mathbf{y}}$ is not efficient. By Lemma 1 , there is an efficient process $\widetilde{\mathbf{z}}$ such that $\widehat{\mathbf{z}} \geq \widehat{\mathbf{y}}$ and $\mathbf{z} \leq \mathbf{y}$, with one of these inequalities being actually strict. Let

$$
\mathbf{u}=(1-\alpha) \mathbf{L t}+\alpha \mathbf{L} \mathbf{s},
$$

and

$$
\widehat{\mathbf{u}}=(1-\alpha) \mathbf{N t}+\alpha \mathbf{N s}
$$

for $\alpha<0$. If $\alpha$ is very small, $(1-\alpha) \mathrm{t}+\alpha \mathrm{s} \geq 0$ and so

$$
\breve{\mathbf{u}}=[-\mathbf{u}, \widehat{\mathbf{u}}]=[-\mathbf{L}((1-\alpha) \mathbf{t}+\alpha \mathbf{s}), \mathbf{N}((1-\alpha) \mathbf{t}+\alpha \mathbf{s})]
$$


is a global activity. Let $\beta=-(\alpha /(1-\alpha))$. Then $0<\beta<1$ and

$$
(1-\beta)[-\mathbf{u}, \widehat{\mathbf{u}}]+\beta[-\mathbf{z}, \widehat{\mathbf{z}}]=[-((1-\beta) \mathbf{u}+\beta \mathbf{z}),(1-\beta) \widehat{\mathbf{u}}+\beta \widehat{\mathbf{z}}]
$$

is also a global process. But

$$
(1-\beta) \mathbf{u}+\beta \mathbf{z} \stackrel{\sim}{=} \mathbf{t}-\beta(\mathbf{y}-\mathbf{z}) \leq \mathbf{L t},
$$

and

$$
\begin{aligned}
(1-\beta) \widehat{\mathbf{u}}+\beta \widehat{\mathbf{z}} & =\frac{1}{1-\alpha}((1-\alpha) \mathbf{N t}+\alpha \mathbf{N s})+\beta \widehat{\mathbf{z}} \\
& =\mathbf{N t}+\frac{\alpha}{1-\alpha} \mathbf{N s}+\beta \widehat{\mathbf{z}} \\
& =\mathbf{N} \mathbf{t}-\beta \mathbf{N} \mathbf{s}+\beta \widehat{\mathbf{z}} \\
& =\mathbf{N t}+\beta(\widehat{\mathbf{z}}-\mathbf{N s}) \\
& =\mathbf{N t}+\beta(\widehat{\mathbf{z}}-\widehat{\mathbf{y}}) \\
& \geq \mathbf{N t} .
\end{aligned}
$$

Now, since

$$
\left(\begin{array}{c}
-(\mathbf{L t}-\beta(\mathbf{y}-\mathbf{z})) \\
\mathbf{N t}+\beta(\widehat{\mathbf{z}}-\widehat{\mathbf{y}})
\end{array}\right) \geq\left(\begin{array}{c}
-\mathbf{L t} \\
\mathbf{N t}
\end{array}\right)
$$

the assumption that $\widetilde{\mathbf{y}}$ is not efficient implies that

$$
\begin{aligned}
\left(\begin{array}{c}
-[\mathbf{L t}-\beta(\mathbf{y}-\mathbf{z})] \\
\mathbf{N t}+\beta(\widehat{\mathbf{z}}-\widehat{\mathbf{y}})
\end{array}\right) & \geq\left(\begin{array}{c}
-\mathbf{L t} \\
\mathbf{N t}
\end{array}\right) \\
& =\breve{\mathbf{x}} .
\end{aligned}
$$

But this is impossible because $\widetilde{\mathbf{x}}$ was supposed to be efficient.

The weight of the Non-Substitution Theorem falls mainly upon two assumptions about the techniques that can be used to produce a certain kind of good. The first assumption is that there is an upper bound to efficiency in the sense that, for the production of any fixed amount of output of kind $h$, the types of the ingredients cannot be changed, and there is also only one way of making optimal use of these ingredients. The second one is that an additional use of inputs to produce a certain amount of good $h, \bar{y}_{h h}$, even if wasteful, requires an additional use of labor power (labor-time). I will discuss these assumptions in what follows.

The gist of the first assumption is not merely that there are no alternative techniques (a typical supposition in Leontief productive structures), but also that there is an upper bound to efficiency in the sense that, for the production of any fixed amount of output of kind $h$, the ingredients cannot be changed and, moreover, there is only one way of making optimal use of these ingredients. A consequence of this thesis is that there is an optimal combination of inputsoutputs, so that any other use of the same ingredients to produce the same 
amount of the good must be inferior to this combination. In other words, for any given amount $\bar{x}_{h h}$ of good $h$ there is a vector of material inputs $\underline{\mathrm{x}}_{h}^{*}$ such that, for every process $\widetilde{\mathbf{y}}_{h}$ in $X_{h}$ with $\bar{y}_{h h}=\bar{x}_{h h}, \underline{\mathbf{x}}_{h}^{*} \leq \underline{\mathbf{y}}_{h}$. What this means is that it is impossible to save in the use of some input beyond the saving made by combination $\underline{\mathbf{x}}_{h}^{*}$, even if some other input is more intensively used. For instance, you cannot produce more shoe soles out of a given piece of leather even if you spend more cutters. On the other hand, you cannot save cutters in the production of the soles out of the same piece beyond a certain point. Thus, even though it is an idealization and false in some cases (in productive structures with alternative techniques), the thesis has a clear economic meaning and is not at all farfetched. The productive structures where it holds will be called "rigid".

The second assumption is also natural and expresses that in order to move machinery and raw materials labor power must be applied. For instance, the optimal use of tractors to sow a given amount of wheat may require a certain number of hours; a less efficient use requires more hours, but then it also requires more labor-time. This is a sort of generalization of the "no free lunch" usual assumption, which sometimes is violated in Leontief productive structures. This violation arises whenever it is possible, in a usual Leontief productive structure, to compensate the inefficient use of material inputs by means of a more efficient use of labor. Suppose, in a structure of that type, that processes $\widetilde{\mathbf{x}}$ and $\widetilde{\mathbf{y}}$ are efficient, that they produce the same output $(\overline{\mathbf{x}}=\overline{\mathbf{y}})$ and that $\widetilde{\mathbf{x}}$ uses more material inputs than $\widetilde{\mathbf{y}}(\underline{\mathbf{x}} \geq \underline{\mathbf{y}})$. By the usual Non-Substitution Theorem, there is a finite number of activities $\widetilde{\mathbf{x}}_{1}, \ldots, \widetilde{\mathbf{x}}_{\eta}$, with $\widetilde{\mathbf{x}}_{h} \in X_{h}(h \in H)$ such that $E \subseteq K\left(\widetilde{\mathbf{x}}_{1}, \ldots, \widetilde{\mathbf{x}}_{\eta}\right)$. Let $\mathbf{L}$ be a vector such that the $h$ th component of $\mathbf{L}$ is the labor input of process $\widetilde{\mathbf{x}}_{h}$, and $\mathbf{N}$ be a $\eta \times \eta$ matrix such that the $h$ th column of $\mathbf{L}$ is the vector of netputs of process $\widetilde{\mathbf{x}}_{h}$. Let $\mathbf{s}$ and $\mathbf{t}$ be non-negative vectors with $\widehat{\mathbf{x}}=\mathbf{N s}, \widehat{\mathbf{y}}=\mathbf{N t}, \mathbf{x}=\mathbf{L s}$ and $\mathbf{y}=\mathbf{L t}$. Since $\mathbf{N}$ has a (non-negative) inverse,

$$
\begin{aligned}
\overline{\mathbf{x}}=\overline{\mathbf{y}} \text { and } \underline{\mathbf{x}} \geq \underline{\mathbf{y}} & \Rightarrow \widehat{\mathbf{x}} \leq \widehat{\mathbf{y}} \\
& \Rightarrow \mathrm{Ns} \leq \mathrm{Nt} \\
& \Rightarrow \mathrm{s} \leq \mathrm{t} \\
& \Rightarrow \mathrm{Ls} \leq \mathrm{Lt} \\
& \Rightarrow \mathrm{x} \leq \mathrm{y}^{\circ}
\end{aligned}
$$

Thus, the inefficent use of material resources by $\widetilde{\mathbf{x}}$ implies that it must be more efficient than $\widetilde{\mathbf{y}}$ in the use of labor.

My proof of the Non-Substitution Theorem with heterogeneous labor requires the ruling out of such possibility of compensation. Hence, I will require that the productive systems be "regular" in the following sense.

Definition 4. Let $\mathcal{X}=\left\{X_{1}, \ldots, X_{\eta}\right\}$ be a Leontief productive structure.

(1) $\mathcal{X}$ is rigid iff, for every kind of good $h$ and every fixed amount $\underline{\mathbf{x}}_{h h}$ of this type of good, there is an optimal combination of inputs for the production of $\bar{x}_{h h}$. In other words, for every process $\widetilde{\mathbf{y}}_{h}$ in $X_{h}$ with $\bar{y}_{h h}=\bar{x}_{h h}, \underline{\mathbf{x}}_{h}^{*} \leq \mathbf{y}_{h}$. 
(2) $\mathcal{X}$ is regular iff, for any two processes $\widetilde{\mathbf{x}}$ and $\widetilde{\mathbf{y}}$ whose output is $\bar{x}_{h h}$, a greater use of resources in one of them implies a greater use of labor power as well. In other words, if $\underline{\mathbf{x}} \geq \underline{\mathbf{y}}$ then $\mathbf{x} \geq \mathbf{y}$.

The optimal combination of inputs-outputs $\left[\underline{x}^{*}, \overline{\mathbf{x}}^{*}\right]$ gives rise to an optimal combination of netputs $\widehat{\mathbf{x}}^{*}$ that has the following property.

Lemma 2: Let $\mathcal{X}=\left\{X_{1}, \ldots, X_{\eta}\right\}$ be a rigid Leontief productive structure and let $\left[\underline{\mathbf{x}}_{h}^{*}, \overline{\mathbf{x}}_{h}^{*}\right]$ be the optimal combination of inputs-outpats required to produce good $\bar{x}_{h h}$. Then, for every process $\widetilde{\mathbf{y}}_{h}$ in $X_{h}$ with $\bar{y}_{h h}=\bar{x}_{h h}, \widehat{\mathbf{y}}_{h} \leq \widehat{\mathbf{x}}_{h}^{*}$.

Proof: In a rigid Leontief productive structure, let $[\mathbf{y}, \underline{\mathbf{y}}, \overline{\mathbf{y}}]_{h} \in Y_{h}$ be such that $\bar{y}_{h h}=\bar{x}_{h h}$, and let $\left[\underline{\mathbf{x}}^{*}, \overline{\mathbf{x}}^{*}\right]$ be the optimal combination of inputs-outputs required to produce good $\bar{x}_{h h}$. Since there is no joint production, $\overline{\mathbf{y}}_{h}=\overline{\mathbf{x}}_{h}^{*}$ and it follows that $\underline{\mathbf{x}}_{h}^{*} \leq \underline{\mathbf{y}}_{h}$ and so $-\underline{\mathbf{y}}_{h} \leq-\underline{\mathbf{x}}_{h}^{*}$. This inequality is preserved if we add the same vector at both sides:

$$
\widehat{\mathbf{y}}_{h}=\overline{\mathbf{y}}_{h}-\underline{\mathbf{y}}_{h} \leq \overline{\mathbf{x}}_{h}^{*}-\underline{\mathbf{x}}_{h}^{*}=\widehat{\mathbf{x}}_{h}^{*}
$$

The gist of the present Non-Substitution Theorem is the claim that, in a rigid and regular Leontief economic structure, there is a family of efficient processes such that any other efficient process is a non-negative linear combination of processes in that family. This is the meaning of the following result.

Theorem 2: Let $X^{+}$be the set of global processes with non-negative net outputs of a rigid and regular Leontief productive structure $X$. If $X^{+}$is productive then there exist a finite number of activities $\widetilde{\mathbf{x}}_{1}^{*}, \ldots, \widetilde{\mathbf{x}}_{\eta}^{*}$, with $\widetilde{\mathbf{x}}_{h}^{*} \in X_{h}$ for $h \in H$, such that $E \subseteq K\left(\widetilde{\mathbf{x}}_{1}^{*}, \ldots, \widetilde{\mathbf{x}}_{\eta}^{*}\right)$.

Proof: For any $h \in H$ and any given level $\overline{\mathbf{x}}_{h h}$ of output of kind $h$, say $\bar{x}_{h h}=1$, let $\left[\underline{\mathbf{x}}_{h}^{*}, \overline{\mathbf{x}}_{h}^{*}\right]$ be the optimal combination of inputs-outputs required to produce good $\bar{x}_{h h}$. Since $X^{+}$is productive, this combination may be such that $\bar{x}_{h h}^{*}=$ $\bar{x}_{h h}^{*}-\underline{x}_{h h}^{*}>0$.

By Lemma 1 , for any process $\widetilde{\mathbf{x}}_{h} \in X_{h}$ with $\left[\underline{\mathbf{x}}_{h}, \overline{\mathbf{x}}\right]=\left[\underline{\mathbf{x}}^{*}, \overline{\mathbf{x}}^{*}\right]$ there is an efficient process $\widetilde{\mathbf{x}}_{h}^{*}=\left[\mathbf{x}_{h}^{*}, \underline{\mathbf{x}}_{h}^{*}, \overline{\mathbf{x}}_{h}^{*}\right]$, with $\mathbf{x}_{h}^{*} \leq \mathbf{x}_{h}$. I claim that every efficient process in $X^{+}$is a linear combination of these processes $\widetilde{\mathbf{x}}_{h}^{*}$. Let $\mathbf{L}$ be a $\eta \times \eta$ matrix such that the $h$ th column of $\mathbf{L}$ is the vector of labor inputs of process $\widetilde{\mathbf{x}}_{h}^{*}$, and $\mathbf{N}$ be a $\eta \times \eta$ matrix such that the $h$ th column of $\mathbf{L}$ is the vector of netputs of process $\widetilde{\mathbf{x}}_{h}^{*}$.

Let $\tilde{\mathbf{y}}$ be an arbitrary element of $E$ and $\left\{\tilde{\mathbf{y}}_{h}\right\}$ a decomposition of $\tilde{\mathbf{y}}$ in simple activities such that $\widetilde{\mathbf{y}}=\sum_{h} r_{h} \widetilde{\mathbf{y}}_{h}$ for non-negative numbers $r_{1}, \ldots, r_{h}$. The same argument given in the proof of Theorem 1 establishes that the cone $K\left(\widetilde{\mathbf{y}}_{1}, \ldots, \widetilde{\mathbf{y}}_{\eta}\right)$ is a set of efficient activities and, since $\widetilde{\mathbf{y}}_{k} \in K\left(\widetilde{\mathbf{y}}_{1}, \ldots, \widetilde{\mathbf{y}}_{\eta}\right)$ for every $h$, the basic activities $\widetilde{\mathbf{y}}_{k}$ themselves are efficient. I will show that there is a non-negative vector $\mathbf{t}$ such that $\breve{\mathbf{y}}=[-\mathbf{L t}, \mathbf{N t}]$. 
Let $t_{h}$ be a number such that $\bar{y}_{h h}=t_{h} \bar{x}_{h h}^{*}$, and notice that the optimality of combination $\left[\underline{\mathbf{x}}_{h}^{*}, \overline{\mathbf{x}}_{h}^{*}\right]$ implies that of $t_{h}\left[\widehat{\mathbf{x}}_{h}^{*}, \overline{\mathbf{x}}_{h}^{*}\right]$. It follows, by Lemma 2 , that $\widehat{\mathbf{y}}_{h} \leq t_{h} \widehat{\mathbf{x}}_{h}^{*}$. But $\widehat{\mathbf{y}}_{h} \leq t_{h} \widehat{\mathbf{x}}_{h}^{*}$ implies that $\underline{y}_{h} \geq t_{h} \underline{\mathbf{x}}_{h}^{*}$ and so more labor is needed to move the machinery and prime materials of $\widetilde{\mathbf{y}}_{h}: \mathbf{y}_{h} \geq t_{h} \mathbf{x}_{h}^{*}$. This means that $\widetilde{\mathbf{y}}_{h}$ is not efficient, contradicting the hypothesis.

Hence, $\widehat{\mathbf{y}}_{h}=t_{h} \widehat{\mathbf{x}}_{h}$ and $t_{h} \mathbf{x}_{h}^{*} \leq \mathbf{y}_{h}$. The efficiency of $\widetilde{\mathbf{y}}_{h}$ demands that, actually, $t_{h} \mathbf{x}_{h}^{*}=\mathbf{y}_{h}$ and so $\widetilde{\mathbf{y}}_{h}=t_{h} \widetilde{\mathbf{x}}_{h}$. It follows that $\widetilde{\mathbf{y}}=\sum_{h=1}^{\eta} t_{h} \widetilde{\mathbf{x}}_{h}^{*}$.

\section{Conclusions}

I have, thus, provided a Non-Substitution Theorem for the static case with heterogeneous labor and established a result with important implications for productive structures more general than the usual Leontief one. In particular, the result holds for productive structures in which activities are not necessarily simple, i.e., in which both joint production and heterogeneous labor are allowed. On the other hand, it seems to me that the Non-Substitution Theorem here established paves the way for a reformulation of the Leontief model of the labor theory of value with more than one primary factor. In his review of my book (García de la Sienra 1992), professor Boris Levin (1994, p. 349) said that the Non-Substitution Theorem

Works with no more than one primary factor. The applicability of this theorem even to a linear dynamic process with technological change is questionable. The author considers this situation to be fully explained. The serious economist refers to "causal indeterminacy", the "constant price assumption", "perfect foresight", etc. And, of course, the possibility of increased returns to scale is not addressed at all.

Certainly, in that book, I was considering only the general static case of the labor theory of value and, therefore, I never considered such a situation "to be fully explained". It seems to me that the present result establishes the validity of the Non-Substitution Theorem for the static case of the Leontief model with more than one primary factor. The constant price "assumption" is actually a logical consequence of this theorem: for a proof, see García de la Sienra (1996) and use the fact that both $\mathbf{L}$ and $\mathbf{N}$ have semi-positive inverses. I do not claim that the present result solves the problems that plague the dynamic Leontief models. Quite another matter is whether an analogous result holds also for these models, and still another one is whether such a result could help to solve those problems. But these questions are beyond the scope of the present paper and will be the topic of future research.

\section{References}

Arrow, K. J. (1951). Alternative Proof of the Substitution Theorem for Leontief Models in the General Case. In Koopmans, T. C. (Ed.). Activity Analysis of Production and Allocation. John Wiley \& Sons, New York, pp. 155-164.

García de la Sienra, A. (1992). The Logical Foundations of the Marxian Theory of Value. Kluwer, Dordrecht. 
García de la Sienra, A. (1996). La Medición del Trabajo Abstracto. Economía Mexicana, 5(1), pp. 63-75.

Georgescu-Roegen, N. (1967). Some Properties of a Generalized Leontief Model. In Georgescu-Roegen, N. (Ed.). Analytical Economics. Harvard University Press, Cambridge. pp. 316-337.

Koopmans, T. C. (1951). Alternative Proof of the Substitution Theorem for Leontief Models in the Case of Three Industries. In Koopmans, T.C. (Ed.). Activity Analysis of Production and Allocation. John Wiley \& Sons, New York, pp. 147-154.

Levhari, D. (1965). A Non-Substitution Theorem and Switching of Techniques. Quarterly Journal of Economics, 79(1), pp. 98-105.

Levin, B. (1994), Review of García de la Sienra (1992). Journal of Evolutionary Economics, 4(4), pp. 347-349.

Mirrles, J. A. (1969). The Dynamic Non-Substitution Theorem. Review of Economic Studies, 36, pp. 67-76.

Nikaido, H. (1968). Convex Structures and Economic Theory. Academic Press, New York.

Salvador, N. (1987). Non-Substitution Theorems. In Eatwell, J., M. Milgate and P. Newman (Eds.). The New Palgrave: A Dictionary of Economics. Macmillan, London.

Samuelson, P. A. (1951). Abstract of a Theorem Concerning Substitutability in Open Leontief Models. In Koopmans, T.C. (Ed.). Activity Analysis of Production and Allocation. John Wiley \& Sons, New York, pp. 142-146.

Samuelson, P. A. (1961). A New Theorem on Non-substitution. In Hegeland, H. (Ed.). Money, Growth and Methodology and Other Essays in Economics. Lund, CWK Gleerup, pp 407-423.

Stiglitz, J. E. (1966). The Collected Scientific Papers of Paul A. Samuelson, vol. 1. MIT Press, Cambridge.

Stiglitz, Joseph E. (1970). Non-Substitution Theorems with Durable Capital Goods. Review of Economic Studies, 37(4), pp. 543-552. 